\title{
Whole genome sequencing provides insights into the genetic determinants of invasiveness in Salmonella Dublin
}

\author{
M. MOHAMMED ${ }^{1,2 *}$ AND M. CORMICAN ${ }^{2}$ \\ ${ }^{1}$ Faculty of Science and Technology, Anglia Ruskin University, Cambridge, UK \\ ${ }^{2}$ School of Medicine, National University of Ireland Galway, Galway, Ireland
}

Received 6 August 2015; Final revision 8 February 2016; Accepted 22 February 2016; first published online 21 March 2016

\section{SUMMARY}

Salmonella enterica subsp. enterica serovar Dublin ( $S$. Dublin) is one of the non-typhoidal Salmonella (NTS); however, a relatively high proportion of human infections are associated with invasive disease. We applied whole genome sequencing to representative invasive and non-invasive clinical isolates of $S$. Dublin to determine the genomic variations among them and to investigate the underlying genetic determinants associated with invasiveness in $S$. Dublin. Although no particular genomic variation was found to differentiate in invasive and non-invasive isolates four virulence factors were detected within the genome of all isolates including two different type VI secretion systems (T6SS) encoded on two Salmonella pathogenicity islands

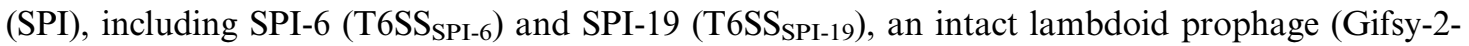
like prophage) that contributes significantly to the virulence and pathogenesis of Salmonella serotypes in addition to a virulence plasmid. These four virulence factors may all contribute to the potential of $S$. Dublin to cause invasive disease in humans.

Key words: Comparative genomics, invasiveness, Salmonella Dublin.

\section{INTRODUCTION}

Non-typhoidal Salmonella (NTS) is a major cause of foodborne disease in humans worldwide. NTS infection is associated with consumption of contaminated food and is typically characterized by self-limiting gastroenteritis. However, some patients with NTS may suffer from bacteraemia. This is more likely in vulnerable groups (very young, very old, immunocompromised) [1]. Certain NTS serovars including $S$. Choleraesuis and $S$. Dublin are associated with higher frequency of occurrence of invasive disease [2]. The invasiveness of $S$. Dublin may be related in part to

\footnotetext{
* Author for correspondence: Dr M. Mohammed, School of Medicine, National University of Ireland-Galway, Galway, Ireland.

(Email: drmanal20@hotmail.com)
}

expression of the Vi (virulence) antigen [3] which is variably expressed in $S$. Dublin and is also commonly found in human-adapted $S$. Typhi.

$S$. Dublin (serotype 1,9,12,[Vi]:g,p:-) is closely related to $S$. Enteritidis (serotype 1,9,12:g,m:-); however, they belong to relatively distinct sequence types (STs) based on multilocus sequence typing (MLST) [4]. Furthermore, S. Dublin and S. Enteritidis differ significantly in their epidemiology. $S$. Dublin is associated with cattle and can be transmitted to humans via contact with infected animals or consumption of contaminated milk and milk products. By contrast, $S$. Enteritidis is associated with poultry and is rarely associated with systemic illness in humans. It has been speculated that extensive genome degradation and pseudogene accumulation within the genome of $S$. Dublin is strongly associated with decreasing the ability of $S$. Dublin to infect a broad range of hosts 
and is involved in bacterial adaptation to certain animal hosts (cattle) [5-7].

Furthermore, $S$. Dublin and $S$. Enteritidis differ in their risk of invasive disease. $S$. Dublin is often associated with invasive disease in humans [8]; however, $S$. Enteritidis is rarely associated with systemic illness in humans. In Ireland, one third of $S$. Dublin clinical isolates received at the National Salmonella, Shigella and Listeria Reference laboratory were isolated from blood and other sterile body fluids. To test whether there is a genomic signal differentiating invasive (blood and other sterile body fluids) and non-invasive (faecal) isolates of $S$. Dublin we determined the whole genome sequencing (WGS) for a set of representative invasive and non-invasive clinical isolates of $S$. Dublin and performed comparative genomics analysis with the closely related $S$. Enteritidis strain P125109 which belongs to phage type 4 (PT4). We also screened the Irish $S$. Dublin isolates for the previously identified pseudogenes [6] that might be involved in host adaptation.

\section{METHODS}

\section{Bacterial strains, genomic DNA extraction and genome sequencing}

A set of invasive and non-invasive $S$. Dublin clinical isolates from Ireland (isolated during 2010-2013) were selected for WGS. Isolates included 11 isolates from human blood and other normally sterile body sites in addition to five gastroenteritis isolates for comparison. Bacterial isolates were cultured on nutrient agar media and incubated overnight at $37^{\circ} \mathrm{C}$. DNA was extracted using QIAamp DNA Mini kit (Qiagen, USA) according to the manufacturer's instructions. WGS was performed using an Illumina MiSeq (Illumina Inc., USA) on $100 \mathrm{bp}$ paired-end (PE) libraries.

Both invasive and non-invasive isolates were tested for the presence of the Vi antigen by slide agglutination using Salmonella Vi antisera.

\section{Sequence data quality control}

The quality of PE Illumina sequence data for each isolate was evaluated using the FastQC toolkit (http:// www.bioinformatics.babraham.ac.uk/projects/fastqc/). Adapter sequences were trimmed and low-quality reads were removed using the ea-utils package (https://code.google.com/p/ea-utils/).
Read mapping, single nucleotide polymorphism (SNP) calling and construction of the phylogenetic tree of the $S$. Dublin sample set

Sequence reads from each isolate were mapped against the reference genome of $S$. Dublin strain CT_02021853 along with its associated plasmid (pCT02021853_74) using Burrows- Wheeler Aligner [9]. Genomic variants including SNPs and insertions and deletions (indels) were identified using Samtools mpileup [10] and filtered with a minimum mapping quality of 60 . SNPs were compared against the reference $S$. Dublin strain CT_02021853 and a maximum-likelihood phylogeny of the isolates was constructed using MEGA v. 6 software [11]. Selection of the best-fit model for nucleotide substitution was performed with jModelTest [12].

\section{S. Dublin genome assembly}

PE reads were de novo assembled using Velvet [13]. The parameters $(k$-mer length, expected coverage, coverage cut-off and insert length) were optimized to obtain the highest N50 value and the best possible assembly. Generated multi-contig draft genomes for each isolate were annotated with the help of the rapid annotation using subsystem technology (RAST) system [14].

\section{Comparative genomic analysis and pseudogene analysis}

Comparative genomic analysis with the reference genome of $S$. Enteritidis strain P125109 and the draft genomes of $S$. Dublin Irish clinical isolates was performed using the Artemis Comparison Tool (ACT) (https://www.sanger.ac.uk/resources/software/act/) in order to identify genes and genomic regions that are specific to $S$. Enteritidis and absent from $S$. Dublin in addition to genes and genomic regions that are specific to $S$. Dublin and absent from $S$. Enteritidis.

We also screened $S$. Dublin isolates for the seven inactive pseudogenes identified previously by microarray analysis [6] that might be involved in adaptation of $S$. Dublin to cattle.

\section{RESULTS}

\section{Phylogenetic relationship between $S$. Dublin isolates}

The phylogenetic relationship between the $S$. Dublin clinical isolates based on SNPs determined by mapping sequences against the genome of reference $S$. Dublin strain CT_02021853 showed that SNPs were found to be randomly distributed around the $S$. Dublin chromosome. 


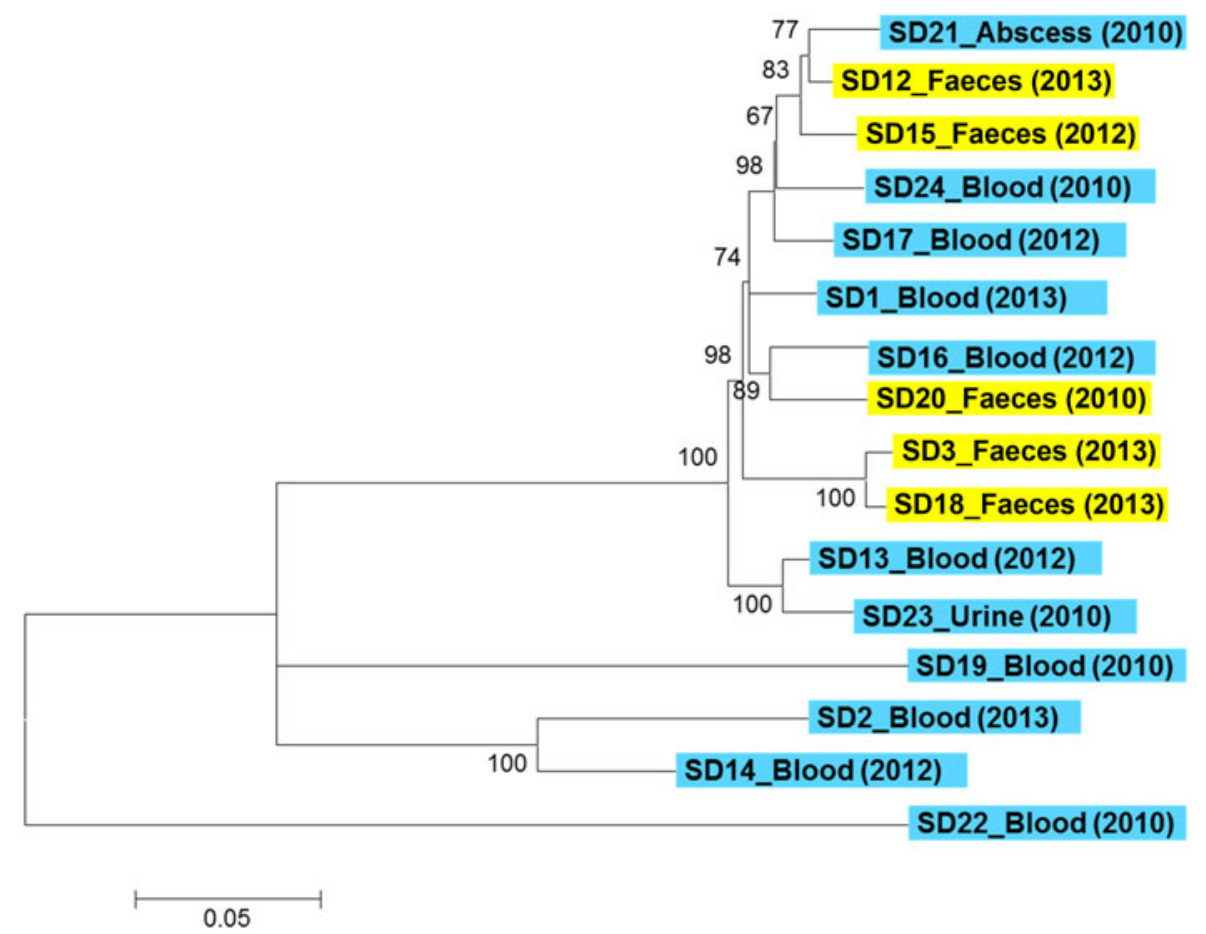

Fig. 1. Phylogenetic tree of Salmonella Dublin isolates based on single nucleotide polymorphisms determined from whole genome sequence. Invasive isolates (highlighted in light blue) and faecal isolates (highlighted in yellow) are intermixed. The generalized time-reversible model of nucleotide substitution for each position was used.

Invasive isolates showed higher genetic divergence (5-333 SNPs) compared to the non-invasive isolates (4-30 SNPs); however, invasive and faecal isolates were intermixed (Fig. 1). SNPs within the chromosome of the $S$. Dublin isolates used in this study are provided in Supplementary Table S1.

\section{Specific genes and genomic regions for $S$. Dublin isolates}

All $S$. Dublin isolates including invasive and gastroenteritis isolates harbour two different types of type VI secretion systems (T6SS). They are encoded in two Salmonella pathogenicity islands (SPI) including SPI-6 (T6SS SPI-19 (T6SS SPI-19, gene range SeD_A1212-SeD_ A1243). Interestingly, T6SS $\mathrm{SPI}_{\mathrm{S}-6}$ and $\mathrm{T}_{6 \mathrm{SS}} \mathrm{SPI-19}$ share very limited homology with each other.

T6SS $_{\text {SPI-6 }}$ presents in all $S$. Dublin clinical isolates including invasive and gastroenteritis isolates in addition to other virulent Salmonella serovars including $S$. Typhi, $S$. Paratyphi A, $S$. Typhimurium and $S$. Choleraesuis (Table 1, Fig. 2) but it is absent from $S$. Enteritidis PT4.

Two virulence genes within $\mathrm{T}_{6 \mathrm{SS}} \mathrm{SPI}-6 ; \quad s c i R$ (STM0284) and sciS (STM0285), encoding the Shiga- like toxin A subunit and putative inner membrane protein, respectively, were present in all $S$. Dublin isolates sequenced in this study.

T6SS $_{\text {SPI-19 }}$ presents in all $S$. Dublin clinical isolates and other host-restricted serovars including $S$. Gallinarum and $S$. Pullorum (Table 1, Fig. 3). However, SPI-19 is degraded in $S$. Enteritidis PT4.

The draft genome of all $S$. Dublin isolates harbours an intact lambdoid prophage (Gifsy-2-like prophage) as illustrated in Figure 4. Gifsy-2-like prophage is degraded in $S$. Enteritidis PT4. Intact Gifsy-2 is also detected in $S$. Typhimurium and $S$. Newport.

Gifsy-2 prophage encodes several virulence factors including sodCI (STM1044), sseI (STM1051) and $\operatorname{gtg} E$ (STM1055) that were found within all $S$. Dublin isolates. Gifsy-2 also encodes $\operatorname{grv} A$; a unique avirulence gene in a wild-type situation; however, a non-synonymous mutation detected in grvA of $S$. Dublin isolates had changed one amino acid (from polar hydrophilic arginine to non-polar hydrophobic tryptophan) within GrvA protein (Arg53-Trp) that might be associated with loss of its antivirulence function, subsequently increasing bacterial virulence.

Interestingly, the gene encoding the Gifsy-2 prophage attachment and invasion protein (STM1043) was found in all $S$. Dublin Irish strains including 
Table 1. Distribution of T6SS loci in Salmonella serotypes

\begin{tabular}{llll}
\hline \hline Serotype & Accession number & \multicolumn{2}{l}{ T6SS $_{\text {SPI-6 }}$ T6SS $_{\text {SPI-19 }}$} \\
\hline Dublin & CP001144 & + & + \\
Enteritidis & AM933172 & - & - \\
Typhimurium* & AE006468 & + & - \\
Typhimurium & FN424405 & + & - \\
Choleraesuis & CP007639 & + & - \\
Typhi & AL513382 & + & - \\
Paratyphi A & CP000026 & + & - \\
Paratyphi B $:$ & CP000886 & - & - \\
Paratyphi C & CP000857 & + & - \\
Kentucky & ABAK02000001 & + & - \\
Newport & CP001113 & + & - \\
Heidelberg & CP005995 & + & - \\
Schwarzengrund & CP001127 & + & - \\
Tennessee & CP007505 & + & - \\
Infantis & NZ_CM001274 & + & + \\
Wetervreden & FR775192, & + & + \\
& FR775207 & & \\
Virchow & ABFH02000001, & - & - \\
& ABFH02000001 & & \\
Javiana & CP004027 & - & - \\
Gallinarum & AM933173 & - & + \\
Pullorum & CP006575 & - & + \\
Agona & CP001138 & - & + \\
Montevideo & CP007222 & + & - \\
Anatum & CP007211 & + & - \\
Thompson & CP006717 & + & - \\
Cubana & CP006055 & + & - \\
\hline \hline
\end{tabular}

* Salmonella Typhimurium strain LT2 (laboratory adapted strain).

$\dagger$ Salmonella Typhimurium strain D23580 (invasive strain). $\$$ Salmonella Paratyphi B strain SPB7 (d-tartrate-fermenting variant; $\mathrm{dT}+$ ).

invasive and gastroenteritis isolates; however, it appears to be subject to partial deletion in $S$. Enteritidis PT4.

All $S$. Dublin isolates including invasive and non-invasive isolates harbour an accessory genome that is closely related to the virulence plasmid (pCT02021853_74) of $S$. Dublin strain CT_02021853 with only 14-22 variants difference. This plasmid is exclusive to $S$. Dublin and is not harboured by other Salmonella serotypes.

No acquired antimicrobial resistance genes were detected within the plasmid or the chromosome of all clinical isolates sequenced here. However, an isolate from faeces (SD12) and another isolate from a psoas abscess (SD21) were resistant to quinolone antimicrobial agents including nalidixic acid as a result of a single non-synonymous mutation within the housekeeping gene $(g y r A)$ that changed the amino acid serine (S) at position 83 to phenylalanine $(F)$ in the DNA gyrase subunit A protein.

The Vi antigen was absent from all $S$. Dublin isolates and the Vi-antigen-encoding genes were absent from the draft genome of $S$. Dublin including invasive isolates. However, the invasion gene $(\operatorname{pag} N)$ was detected in both invasive and gastroenteritis $S$. Dublin isolates.

\section{Specific genes and genomic regions for $\boldsymbol{S}$. Enteritidis}

Forty genes present in $S$. Enteritidis appear to be entirely absent from $S$. Dublin isolates (Table 2). Two genes (SEN2420 and SEN3943) encode a putative exported protein and a conserved hypothetical protein, respectively. The remaining 38 genes were grouped into five genomic regions: SE_Reg1 (SEN0083-SEN0085) encodes two probable secreted proteins and a putative sulfatase, SE_Reg2 (SEN1379-SEN1396) is a part of phage SE14 encoding several phage-related proteins, SE_Reg3 (SEN1432-SEN1436) corresponds to a genomic island named ROD13 and includes genes encoding proteins involved in sugar transport and hexonate uptake, SE_Reg4 (SEN1503-1506) corresponds to another genomic island known as ROD14 encoding regulatory proteins. In addition, another genomic region, SE_Reg5 (SEN1936-SEN1940, SEN1944-SEN1945 and SEN1959), which is absent from $S$. Dublin Irish isolates, encodes several phage-related proteins including terminases and capsid and membrane proteins.

\section{Pseudogene analysis}

The draft genomes of the $S$. Dublin clinical isolates were screened for the previously identified seven coding sequences (CDS) that appear to encode active genes in the broad host range serovar $S$. Enteritidis PT4, but are inactive (pseudogenes) in the seven Uruguayan $S$. Dublin clinical isolates [6] as determined by microarray analysis. Interestingly, we found that these seven CDS are also inactive in all of the Irish clinical isolates of $S$. Dublin sequenced in this study and also in the other host-restricted serovars including the poultry-adapted $S$. Gallinarum and the swine-adapted $S$. Cholerasuis (Table 3).

These CDS correspond to a putative transport protein (SEN0042), two hypothetical proteins (SEN0784 and SEN2783), the gene encoding a probable glucarate dehydratase 2 (SEN2806), the gene encoding the outer membrane usher protein LpfC (SEN3461) and the gene 


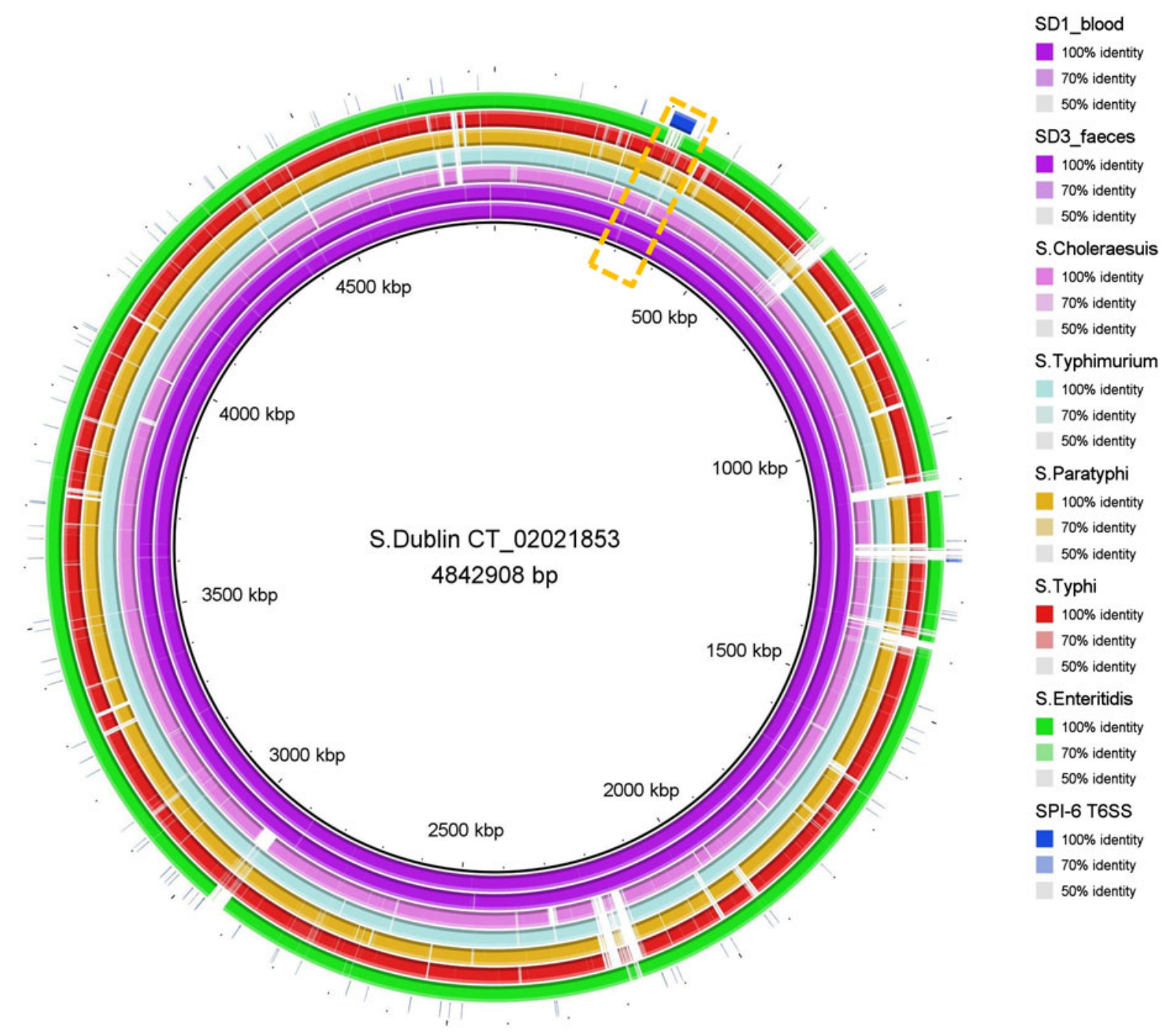

Fig. 2. Complete genome alignment of different Salmonella serovars (including the draft genome of representatives of clinical Irish isolates of $S$. Dublin sequenced in this study) generated using BRIG [27]. S. Dublin strain CT_02021853 genome is used as a reference. The gene cluster of SPI-6 T6SS (gene range SeD_A0289-SeD_A0326) is also included in the alignment. The genome of clinical $S$. Dublin isolates (including invasive and gastroenteritis isolates) and other invasive serovars including $S$. Choleraesuis, $S$. Typhimurium, $S$. Typhi and $S$. Paratyphi A harbours SPI-6 T6SS while it is absent from $S$. Enteritidis.

encoding probable phosphotransferase system permease (SEN3672). In addition $m g l A$ (SEN2182) appear to be inactive in all host-adapted typhoidal Salmonella serovars including the human-adapted $S$. Typhi and $S$. Paratyphi A (Table 3).

A gene of special interest in clinical microbiology, designated apeE, that encodes esterase was found to be inactive (as a result of frameshift mutation) in all $S$. Dublin clinical isolates but is active in other Salmonella serovars including Enteritdis, Typhimurium and Typhi. This finding can explain the failure to detect $S$. Dublin on Aes Laboratoire Salmonella Agar Plate (ASAP) [15]. Although apeE is also absent from some other Salmonella serovars including Choleraesuis, Newport, Agona and Heidelberg these serovars harbour a gene closely related to apeE ( $\sim 98 \%$ identity) that codes lipase which has the ability to hydrolyse a variety of esters, therefore ASAP chromogenic medium will be suitable for their detection. However, the gene coding lipase was also inactive in all $S$. Dublin clinical isolates.

\section{DISCUSSION}

Human infection with $S$. Dublin represents a significant public health problem. In Ireland, an outbreak 


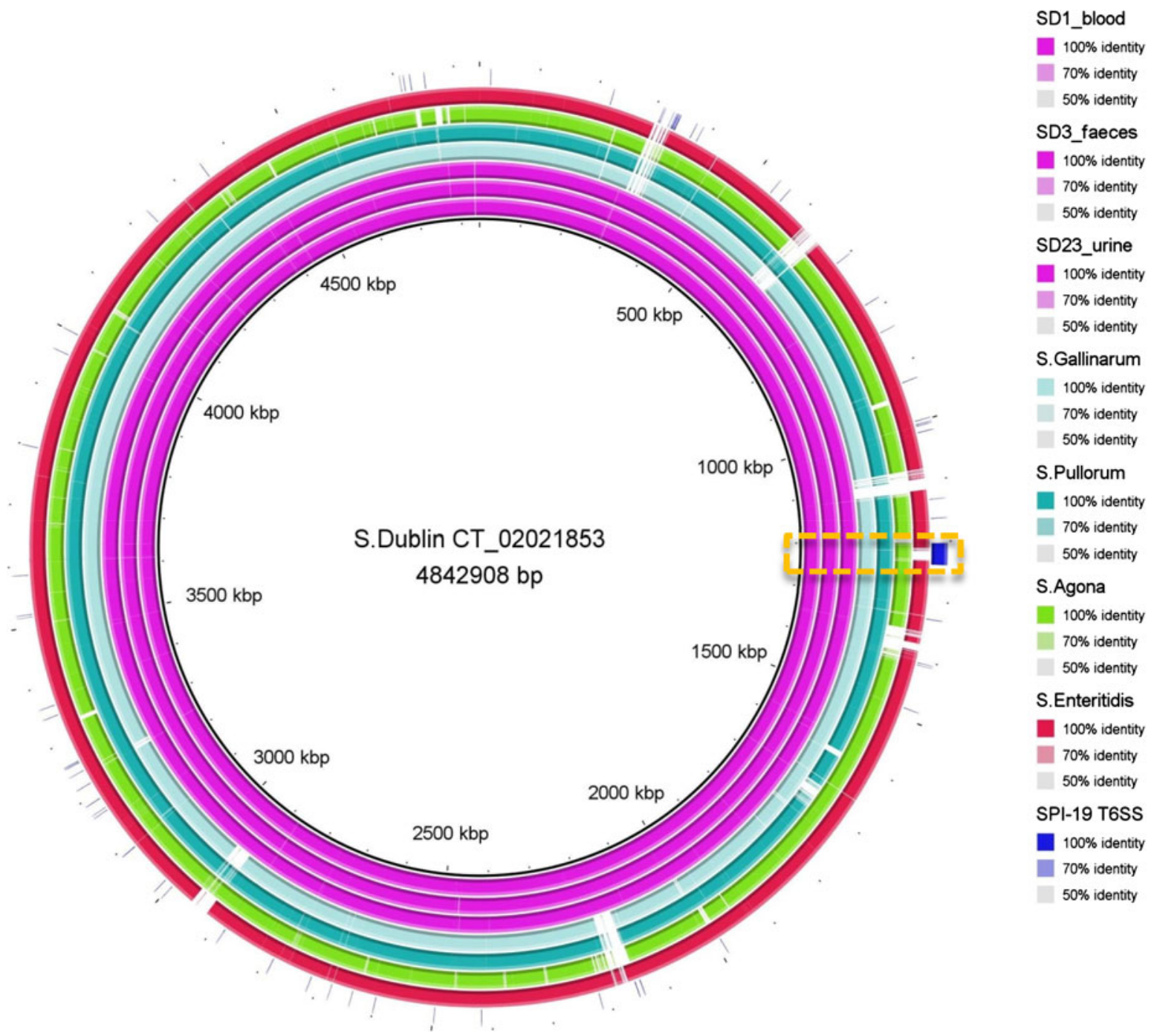

Fig. 3. Complete genome alignment of different Salmonella serovars (including the draft genome of representatives of clinical Irish isolates of $S$. Dublin sequenced in this study) generated using BRIG [27]. S. Dublin strain CT_02021853 genome is used as a reference. The gene cluster of SPI-19 T6SS (gene range SeD_A1212-SeD_A1243) is also included in the alignment. The genome of $S$. Dublin clinical isolates (including invasive and gastroenteritis isolates), $S$. Agona and the poultry-adapted serovars including $S$. Gallinarum and $S$. Pullorum harbours SPI-19T6SS while it is absent from $S$. Enteritidis.

of $S$. Dublin gastroenteritis confirmed by WGS was reported in October-November 2013 [16]. Although all isolates associated with this specific outbreak were from faecal samples prior to the outbreak, one third of $S$. Dublin clinical isolates received at the National Salmonella, Shigella and Listeria Reference laboratory were isolated from blood and other sterile body fluids.

Here we applied WGS to a new set of $S$. Dublin clinical isolates including invasive and gastroenteritis isolates and performed comparative genomic analysis in $S$. Dublin clinical isolates and the reference $S$. Enteritidis PT4 to investigate the underlying bacterial genetic determinants that might be associated with virulence in $S$. Dublin.

Two different T6SS encoded on two pathogenicity islands, $\mathrm{T}_{6 S S_{\mathrm{SPI}-6}}$ and $\mathrm{T} 6 \mathrm{SS}_{\mathrm{SPI}-19}$, are present in all $S$. Dublin isolates described in this study but absent from $S$. Enteritidis PT4. Interestingly, T6SS $\mathrm{SPI}_{\mathrm{SP}}$ and T6SS $S_{\text {SPI-19 }}$ share very limited homology with each other.

Experimental studies have showed the crucial role of $\mathrm{T}_{6 \mathrm{SS}} \mathrm{SPI}_{\text {-6 }}$ in the systemic spread of $S$. Typhimurium [17]. Interestingly, two virulence genes within $\mathrm{T}_{6 \mathrm{SS}}$ SPI-6; sciR (STM0284) and sciS (STM0285) which encode the Shiga-like toxin A subunit and 


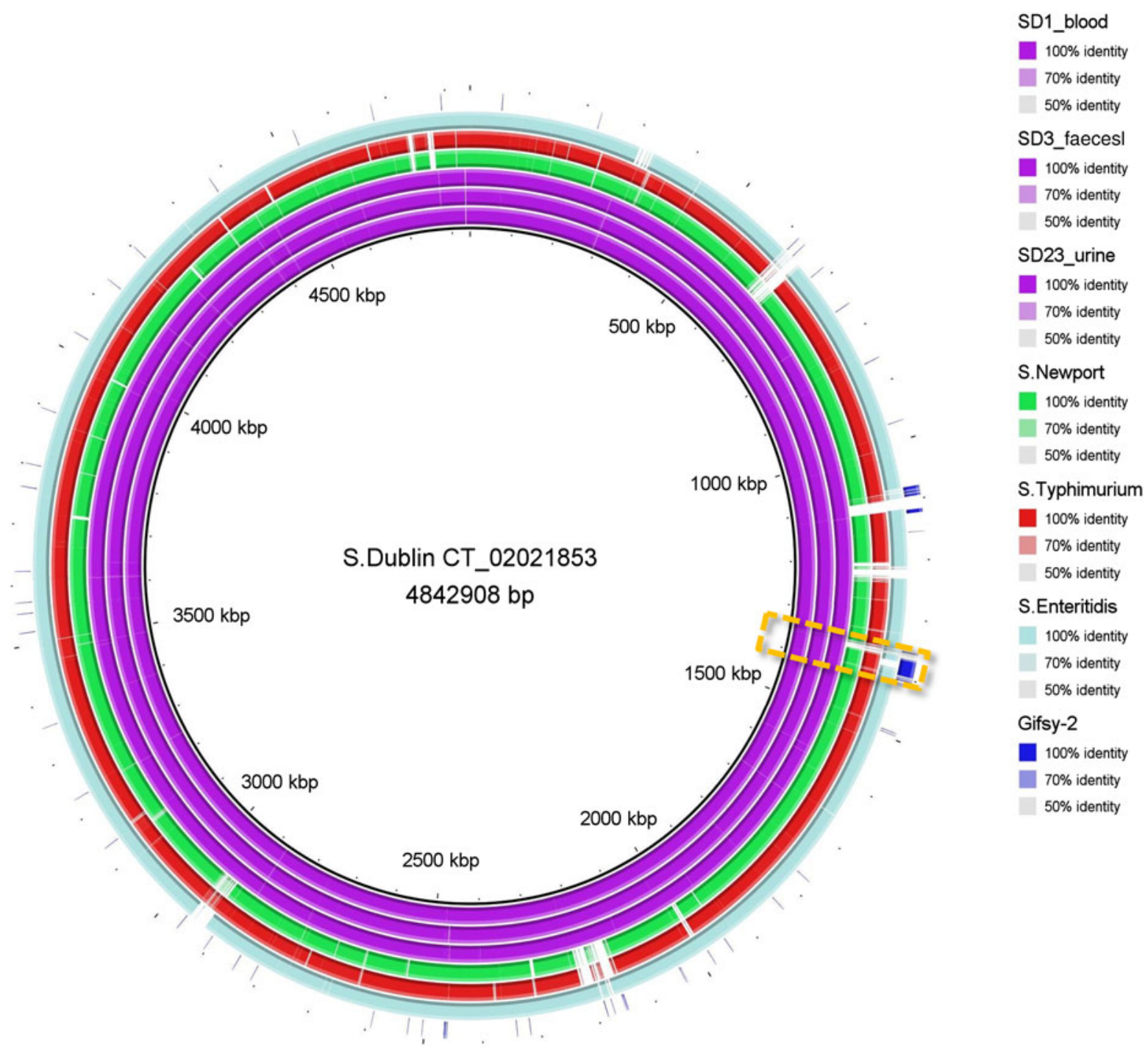

Fig. 4. Complete genome alignment of different Salmonella serovars (including the draft genome of representatives of clinical Irish isolates of S. Dublin sequenced in this study) generated using BRIG [27]. S. Dublin strain CT_02021853 genome is used as a reference. Prophage Gifsy-2 genome is also included in the alignment. The genome of clinical $S$. Dublin isolates (including invasive and gastroenteritis isolates), $S$. Typhimurium and $S$. Newport harbours Gifsy-2 prophage while Gifsy-2 is defective in $S$. Enteritidis PT4.

putative inner membrane protein, respectively, were absent from $S$. Enteritidis PT4 but present in all $S$. Dublin isolates. We also found that $\mathrm{T}_{6 \mathrm{SS}} \mathrm{SPI}-6$ is present in other virulent Salmonella serovars including Typhi, Paratyphi A, Choleraesuis and Typhimurium suggesting that $\mathrm{T}_{6 \mathrm{SS}} \mathrm{SPI}_{-6}$ contributes to the ability of Salmonella to cause invasive disease.

T6SS $_{\text {SPI-19 }}$ was also carried by all $S$. Dublin clinical isolates. Interestingly, $\mathrm{T} \mathrm{SS}_{\mathrm{SPI}-19}$ is present in other host-restricted serovars including poultry-associated $S$. Gallinarum and $S$. Pullorum suggesting its role in adaptation to specific animals [5, 18]. However, SPI-19 is subject to internal deletion in the broad host range serovar Enteritidis PT4. It has been reported that loss of both T6SSs in $S$. Enteritidis appears to be correlated to adaptation to a wide host range [5].

All $S$. Dublin clinical isolates are lysogenic for a lambdoid prophage (Gifsy-2-like prophage), which is defective in $S$. Enteritidis PT4 [19]. Gifsy-2 contributes significantly to the virulence and pathogenesis of Salmonella serotypes [20] as it encodes several virulence factors including sodCI (STM1044), sseI (STM1051) and $g \operatorname{tg} E$ (STM1055) [21]. Gifsy-2-like prophage might therefore be associated with the propensity of $S$. Dublin to cause invasive disease. 
Table 2. Single genes and regions (Reg) that are exclusive to Salmonella Enteritidis PT4 strains but are absent from Salmonella Dublin strains

\begin{tabular}{ll}
\hline \hline $\begin{array}{l}\text { Present in Enteritidis PT4 but absent } \\
\text { from all Dublin }\end{array}$ & Function/gene prediction \\
\hline SE_Reg1 (SEN0083-SEN0085) & Two probable secreted proteins and a putative sulfatase \\
SE_Reg2 (SEN1379-SEN1396) & Part of phage SE14 encoding several phage-related proteins \\
SE_Reg3 (SEN1432-SEN1436) & $\begin{array}{c}\text { Part of ROD13 genomic island encoding proteins involved in sugar transport } \\
\text { and hexonate uptake }\end{array}$ \\
SE_Reg4 (SEN1503-1506) & Part of ROD14 encoding regulatory proteins \\
SE_Reg5 (SEN1936-SEN1940, & Encode several phage-related proteins including terminases and capsid and \\
SEN1944-SEN1945 and SEN1959) & membrane proteins \\
SEN2420 & Putative exported protein \\
SEN3943 & Conserved hypothetical protein \\
\hline \hline
\end{tabular}

Table 3. List of the seven coding sequences that are predicted to be active genes in Salmonella Enteritidis PT4 but inactive (pseudogenes) in Salmonella Dublin isolates

\begin{tabular}{|c|c|c|c|c|c|c|c|}
\hline Gene & Prediction & $\begin{array}{l}\text { Enteritidis } \\
\text { (PT4) }\end{array}$ & $\begin{array}{l}\text { Dublin } \\
\text { (Irish isolates) }\end{array}$ & $\begin{array}{l}\text { Gallinarum } \\
(287 / 91)\end{array}$ & $\begin{array}{l}\text { Cholerasuis } \\
\text { (C500) }\end{array}$ & $\begin{array}{l}\text { Typhi } \\
\text { (CT18) }\end{array}$ & $\begin{array}{l}\text { Paratyphi A } \\
\text { (ATCC 9150) }\end{array}$ \\
\hline SEN0042 & $\begin{array}{l}\text { Putative transport } \\
\text { protein }\end{array}$ & Active & Pseudo & Pseudo & Pseudo & Active & Active \\
\hline SEN0784 & Hypothetical protein & Active & Pseudo & Pseudo & Pseudo & Absent & Absent \\
\hline SEN2182* & $\begin{array}{l}m g l A \text {, galactoside } \\
\text { transport ATP binding } \\
\text { protein }\end{array}$ & Active & Pseudo & Pseudo & Pseudo & Pseudo & Pseudo \\
\hline SEN2783 & Hypothetical protein & Active & Pseudo & Pseudo & Pseudo & Absent & Absent \\
\hline SEN2806 & $\begin{array}{l}\text { Probable glucarate } \\
\text { dehydratase } 2\end{array}$ & Active & Pseudo & Pseudo & Pseudo & Active & Partial deletion \\
\hline SEN3461 & $\begin{array}{l}\text { Outer membrane usher } \\
\text { protein LpfC }\end{array}$ & Active & Pseudo & Pseudo & Pseudo & Absent & Absent \\
\hline SEN3672 & $\begin{array}{l}\text { Probable } \\
\text { phosphotransferase } \\
\text { system permease }\end{array}$ & Active & Pseudo & Pseudo & Pseudo & Active & Partial deletion \\
\hline
\end{tabular}

A unique antivirulence gene, $\operatorname{grv} A$, is carried on Gifsy-2. It decreases bacterial pathogenicity in its wild-type situation playing a role in optimizing Salmonella virulence [22]. However, the nonsynonymous mutation detected in grvA of $S$. Dublin isolates had changed one amino acid (polar, hydrophilic arginine to non-polar hydrophobic tryptophan) within the GrvA protein (Arg53-Trp). We postulate that this may be associated with loss of its antivirulence function.

Interestingly, the gene encoding the Gifsy- 2 prophage attachment and invasion protein (STM1043) was found in all $S$. Dublin strains; however, it was subjected to partial deletion in $S$. Enteritidis PT4.

Although the massive accumulation of pseudogenes (especially genes involved in metabolic pathways) in
$S$. Dublin has been reported to be associated with adaptation to cattle and invasiveness in humans [5], it can also be associated with false-negative results in detection of $S$. Dublin at clinical laboratories when chromogenic medium (ASAP) is used since the apeE gene encodes esterase [23], responsible for bacterial ability to hydrolyse ester substrate methyl umbelliferyl caprylate within the chromogenic medium, which was found to be inactive in all $S$. Dublin isolates.

Therefore, $S$. Dublin is not able to produce ApeE esterase during overnight incubation and no expected pink to purple colonies are produced [15]. The unique biochemical features of $S$. Dublin [24] can delay bacterial identification by clinical laboratories where prompt identification is crucial since $S$. Dublin can be associated with invasiveness and systemic illness. 
We looked at the genetic differences in invasive and gastroenteritis isolates of $S$. Dublin, but no particular genetic determinants were found to differentiate among isolates. Phylogenetic analysis based on SNPs determined from WGS showed that invasive and faecal isolates are intermixed and SNPs were found to be randomly distributed around the $S$. Dublin chromosome. Furthermore, a plasmid that is closely related to the virulence plasmid (pCT02021853_74) of $S$. Dublin strain CT_02021853 was detected in all $S$. Dublin clinical isolates. This plasmid is limited to $S$. Dublin and has not been detected in other Salmonella serotypes. However, this plasmid carries virulence genes that proved to be associated with causing systemic illness [25].

Although the invasiveness of $S$. Dublin has been considered as related to expression of the Vi antigen, the encoding genes [26] were absent from all $S$. Dublin clinical isolates including invasive isolates.

Interestingly, the genome of all $S$. Dublin clinical 'invasive and faecal' isolates sequenced in this study harbours an active invasion gene ( $p a g N)$ which might be associated with the ability to cause invasive illness.

In conclusion, our results reveal several virulence genetic determinants within the genome of $S$. Dublin from Ireland that gives this serovar the potential to cause invasive disease in humans. However, there were no genomic markers that differentiated invasive from gastroenteritis isolates suggesting that host factors are critical in determining the outcome in the individual patient.

Comparison of larger numbers of Salmonella isolates from different geographical and temporal spreads may provide detailed insights into the basis of bacterial virulence and invasiveness.

\section{SUPPLEMENTARY MATERIAL}

For supplementary material accompanying this paper visit http://dx.doi.org/10.1017/S0950268816000492.

\section{ACKNOWLEDGEMENTS}

This research received no specific grant from any funding agency, commercial or not-for-profit sectors.

\section{DECLARATION OF INTEREST}

None.

\section{REFERENCES}

1. Bronowski C, et al. Genomic characterisation of invasive non-typhoidal Salmonella enterica subspecies enterica serovar Bovismorbificans isolates from Malawi. PLoS Neglected Tropical Diseases 2013; 7: e2557.

2. Jones TF, et al. Salmonellosis outcomes differ substantially by serotype. Journal of Infectious Diseases 2008; 198: 109-114.

3. Morris C, et al. Salmonella enterica serovar Dublin strains which are Vi antigen-positive use type IVB pili for bacterial self-association and human intestinal cell entry. Microbial Pathogenesis 2003; 35: 279-284.

4. Achtman M, et al. Multilocus sequence typing as a replacement for serotyping in Salmonella enterica. PLoS Pathogens 2012; 8: e1002776.

5. Langridge GC, et al. Patterns of genome evolution that have accompanied host adaptation in Salmonella. Proceedings of the National Academy of Sciences USA 2015; 112: 863-868.

6. Betancor L, et al. Genomic comparison of the closely related Salmonella enterica serovars Enteritidis and Dublin. Open Microbiology Journal 2012; 6: 5-13.

7. Moran NA, Plague GR. Genomic changes following host restriction in bacteria. Current Opinion in Genetics and Development 2004; 14: 627-633.

8. Fierer J. Invasive Salmonella Dublin infections associated with drinking raw milk. Western Journal of Medicine 1983; 138: 665-669.

9. Li H, Durbin R. Fast and accurate short read alignment with Burrows-Wheeler transform. Bioinformatics 2009; 25: $1754-1760$.

10. Li H, et al. The sequence alignment/map format and SAMtools. Bioinformatics 2009; 25: 2078-2079.

11. Tamura K, et al. MEGA6: Molecular Evolutionary Genetics Analysis version 6.0. Molecular Biology and Evolution 2013; 30: 2725-2729.

12. Posada D. jModelTest: phylogenetic model averaging. Molecular Biology and Evolution 2008; 25: 1253-1256.

13. Zerbino DR, Birney E. Velvet: algorithms for de novo short read assembly using de Bruijn graphs. Genome Research 2008; 18: 821-829.

14. Aziz RK, et al. The RAST Server: rapid annotations using subsystems technology. BMC Genomics 2008; 9: 75.

15. Gray S, et al. Failure to detect Salmonella enterica serovar Dublin on Aes Laboratoire Salmonella Agar Plate. Journal of Clinical Microbiology 2003; 41: 4003.

16. Mohammed M, et al. Whole genome sequencing provides an unambiguous link between Salmonella Dublin outbreak strain and a historical isolate. Epidemiology and Infection 2016; 144: 571-581.

17. Pezoa D, et al. The type VI secretion system encoded in SPI-6 plays a role in gastrointestinal colonization and systemic spread of Salmonella enterica serovar Typhimurium in the chicken. PLoS ONE 2013; 8: e63917.

18. Blondel CJ, et al. The type VI secretion system encoded in Salmonella pathogenicity island 19 is required for Salmonella enterica serotype Gallinarum survival within infected macrophages. Infection and Immunity 2013; 81: 1207-1220. 
19. Matthews TD, et al. Genomic comparison of the closely-related Salmonella enterica serovars Enteritidis, Dublin and Gallinarum. PLoS ONE 2015; 10: e0126883.

20. Figueroa-Bossi N, Bossi L. Inducible prophages contribute to Salmonella virulence in mice. Molecular Microbiology 1999; 33: 167-176.

21. Ho TD, et al. Identification of GtgE, a novel virulence factor encoded on the Gifsy-2 bacteriophage of Salmonella enterica serovar Typhimurium. Journal of Bacteriology 2002; 184: 5234-5239.

22. Ho TD, Slauch JM. Characterization of grvA, an antivirulence gene on the gifsy-2 phage in Salmonella enterica serovar Typhimurium. Journal of Bacteriology 2001; 183: 611-620.

23. Carinato ME, et al. The apeE gene of Salmonella Typhimurium encodes an outer membrane esterase not present in Escherichia coli. Journal of Bacteriology 1998; 180: 3517-3521.

24. Fierer J, Fleming W. Distinctive biochemical features of Salmonella Dublin isolated in California. Journal of Clinical Microbiology 1983; 17: 552-554.

25. Libby SJ, et al. The spv genes on the Salmonella Dublin virulence plasmid are required for severe enteritis and systemic infection in the natural host. Infection and Immunity 1997; 65: 1786-1792.

26. Pulickal AS, et al. Prevalence and genetic analysis of phenotypically Vi- negative Salmonella Typhi isolates in children from Kathmandu, Nepal. Journal of Tropical Pediatrics 2013; 59: 317-320.

27. Alikhan NF, et al. BLAST Ring Image Generator (BRIG): simple prokaryote genome comparisons. BMC Genomics 2011, 12: 402. 